\title{
Reducing the Spurious Oscillations in the Lumped Parameters Model Using Low-Pass Filter
}

\author{
A. R. J.de Araújo, R. C. da Silva and S. Kurokawa
}

\begin{abstract}
Voltages and currents in the transmission line are described by differential equations that are difficult to solve due soil and skin effect that has to be considered for accurate results, but it increases their complexity. Therefore there are some models to study the voltages and currents along in transmission line. The distributed parameters model that transforms the equations in time domain to the frequency domain and once the solutions are obtained, they are converted to time domain using the Inverse Laplace Transform using numerical methods. Another model is named lumped parameters model and it considers the transmission line represented by a $\pi$-circuit cascade and the currents and voltages are described by state equations. In the simulations using the lumped parameters model, it can be observed the presence of spurious oscillations that are independent of the quantity of $\pi$ circuits used and do not represent the real value of the transient. In this work will be projected a passive low-pass filter directly inserted in the lumped parameters model to reduce the spurious oscillations in the simulations, making this model more accurate and reliable for studying the electromagnetic transients in power systems.
\end{abstract}

Keywords - Electromagnetic transients, transmission lines, distributed parameters, lumped parameters, low-pass filter.

\section{INTRODUÇÃO}

$\mathrm{T}$ ENSÕES e correntes em linhas de transmissão são descritas por equações diferenciais que são de difícil resolução devido ao efeitos solo e frequência e que devem ser considerados sobre os parâmetros da linha para uma resposta precisa e confiável. Nesse contexto diversos pesquisadores e cientistas têm dedicado seus esforços para resolução dessas equações. $\mathrm{O}$ primeiro trabalho considerando transitórios eletromagnéticos foi desenvolvido por Lord Kevin em 1854 quando estudava a propagação de ondas em cabos de telecomunição [1]. Heaviside desenvolveu a "Transformada de Heaviside" resultando em equações/soluções nos domínios do tempo e frequência. Essa transformada foi utilizada para obter a resposta transitória em circuitos elétricos representados por parâmetros discretos. Desde 1960 pesquisadores se dedicam no desenvolvimento de modelos de linhas de transmissão para estudo de transitórios eletromagnéticos em sistemas de potência.

H. W. Dommel desenvolveu um dos primeiros modelos para representar linhas de transmissão diretamente no domínio do tempo. Esse modelo foi baseado no método de Bergeron e combina o método numérico trapezoidal, resultando num algoritmo para estudo de transitórios eletromagnéticos

A. R. J. de Araujo, Universidade Estadual Paulista (UNESP), Ilha Solteira, São Paulo, Brasil, anderjusto@yahoo.com.br

R.C.da Silva, Universidade Estadual Paulista (UNESP), Ilha Solteira, São Paulo,Brasil,rcleber@gmail.com

S. Kurokawa, Universidade Estadual Paulista (UNESP), Ilha Solteira, São Paulo,Brasil,kurokawa@dee.feis.unesp.br. em sistemas de potência usando parâmetros discretos ou distribuídos para representação da linha. Este algoritmo é denominado Electromagnetic Transients Program, EMTP [2]. Para estudar a propagação de ondas eletromagnéticas resultantes de processos de chaveamento, a linha de transmissão pode ser representada por uma cascata de circuitos $\pi$ [3-5]. Esse modelo tem como vantagens o fato de considerar cargas não-lineares, perdas pelo efeito corona, além de ser desenvolvido diretamente no domínio do tempo.

A representação de linhas por cascatas de circuito $\pi$ considera que um segmento de linha, cujos parâmetros são distribuídos ao longo de seu comprimento, pode ser representado por elementos discretos de circuito conectados em cascata. $[7,8]$.

Alguns autores [2,6-8] sugerem escrever as correntes e tensões usando equações de estado. As equações de estado são resolvidas usando um método numérico em qualquer linguagem computacional [8-9]. Nesse trabalho será utilizado o método de integração trapezoidal de Heun [9].

Outro modelo proposto para estudar transitórios eletromagnéticos é denominado de Universal Line Model (ULM) [10]. Nesse modelo as equações diferenciais são transformadas em equações algébricas hiperbólicas usando a transformada de Laplace. O modelo considera os parâmetros longitudinais e transversais da linha e pode incluir o efeito da frequência para resultados mais precisos.

As soluções no domínio do tempo são obtidas utilizando a Transformada Inversa de Laplace implementada numericamente [11,12]. Nas simulações obtidas com o modelo a parâmetros discretos, observam-se oscilações numéricas que são denominadas de oscilações espúrias. Essas oscilações apresentam picos de sobretensão que não representam o valor real das tensões e corrente e caso fossem considerados, poderiam causar atuação indevida de sistemas de proteção ou projetos sobrestimados de isoladores, tornando-o mais custosos. As oscilações ocorrem devido à representação da linha pela cascada de circuitos $\pi$ e independem do método numérico utilizado para resolução das equações de estado [9].

Este trabalho analisará as oscilações espúrias presentes nas simulações de uma linha de transmissão monofásica de $100 \mathrm{~km}$ e 100 circuitos $\pi$ em cascata, energizada por uma fonte senoidal de tensão. Será estudado o espectro de frequência das oscilações espúrias para linha representada pelo modelo a parâmetros discretos e será projetado um filtro passivo analógico inserido diretamente no modelo, para a redução das oscilações, fazendo com que o modelo se torne mais preciso e confiável para estudo de transitórios eletromagnéticos em sistemas de potência. 


\section{SOLUÇÕES DAS EQUAÇÕES DIFERENCIAIS DA LINHA DE TRANSMISSÃO}

As equações diferenciais que modelam a linha de transmissão são de difícil resolução no domínio do tempo, mas no domínio da frequência elas se tornam simples equações algébricas hiperbólicas e as soluções são de fácil obtenção. A solução no domínio da frequência é genérica e pode ser aplicada a qualquer condição da linha como, por exemplo, considerar os parâmetros fixos ou dependentes da frequência. A seguir serão apresentados os modelos a parâmetros discretos e distribuídos.

\section{A. MODELO A PARÂMETROS DISTRIBUÍDOS}

Considerando uma linha de transmissão (LT) de comprimento $d$ no domínio da frequência conforme mostra a Fig. 1.

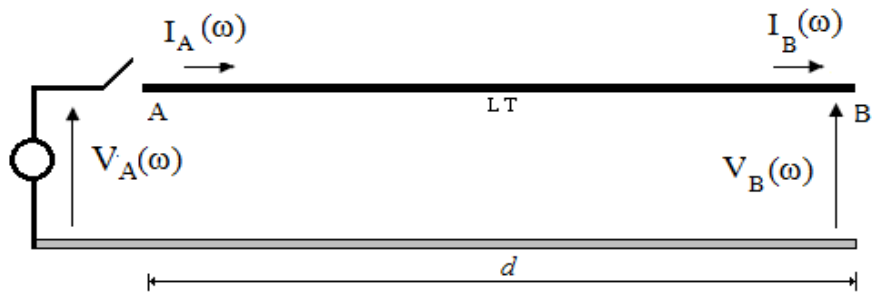

Figura 1. Linha de transmissão de comprimento $d$.

A linha de transmissão (LT) tem uma impedância longitudinal e admitância transversal dadas por (1):

$$
\begin{aligned}
& Z(\omega)=R+j \omega L \\
& Y(\omega)=G+j \omega C
\end{aligned}
$$

Em (1) e (2) os termos R e L são a resistência e indutância longitudinal por unidade de comprimento e os termos $\mathrm{C}$ e $\mathrm{G}$ são as capacitância e condutância transversal por unidade de comprimento. Os termos $\mathrm{I}_{\mathrm{A}}(\omega)$ e $\mathrm{I}_{\mathrm{B}}(\omega)$ são as correntes nos terminais emissor e receptor da $L T$ enquanto que $\mathrm{V}_{\mathrm{A}}(\omega)$ e $\mathrm{V}_{\mathrm{B}}(\omega)$ são as tensões nos respectivos terminais e $\omega$ é a frequência angular. As correntes e tensões no domínio da frequência são dadas por [14]:

$$
\begin{aligned}
& I_{A}(\omega)=Y_{A A}(\omega) V_{A}(\omega)+Y_{A B}(\omega) V_{B}(\omega) \\
& I_{B}(\omega)=Y_{B A}(\omega) V_{A}(\omega)+Y_{B B}(\omega) V_{B}(\omega)
\end{aligned}
$$
por :

Os termos $\mathrm{Y}_{\mathrm{AA}}(\omega), \mathrm{Y}_{\mathrm{AB}}(\omega), \mathrm{Y}_{\mathrm{BA}}(\omega)$ e $\mathrm{Y}_{\mathrm{BB}}(\omega)$ são dados

$$
\begin{array}{ll}
Y_{A A}=\frac{1}{Z_{C}} \operatorname{coth}(\gamma(\omega) d) & Y_{A B}=-\frac{1}{Z_{C}} \operatorname{csch}(\gamma(\omega) d) \\
Y_{B A}=\frac{1}{Z_{C}} \operatorname{coth}(\gamma(\omega) d) & Y_{B B}=-\frac{1}{Z_{C}} \operatorname{csch}(\gamma(\omega) d)
\end{array}
$$

Em (5) $Z_{C}(\omega)$ e $\gamma(\omega)$ são a impedância de surto e a função de propagação respectivamente dadas por :

$$
\begin{aligned}
& Z_{C}(\omega)=\sqrt{\frac{Z(\omega)}{Y(\omega)}} \\
& \gamma(\omega)=\sqrt{Z(\omega) Y(\omega)}
\end{aligned}
$$

A transformação de (3) e (4) no domínio do tempo é difícil de obter devido as integrais de convolução. $\mathrm{O}$ modelo a parâmetros distribuídos considera que os parâmetros da linha são distribuídos ao longo de seu comprimento. Nesse modelo as equações diferenciais são transformadas em equações algébricas hiperbólicas no domínio da frequência. Assim o modelo quando representado por essas equações é denominado por Universal Line Model (ULM) [11]. Uma vez obtidas as soluções no domínio da frequência, utilizando ac transformada Inversa de Laplace implementada numericamente, são obtidas as soluções são obtidas no domínio do tempo. A transformada de Laplace de uma função $\mathrm{f}(t)$ é definida por:

$$
F(s)=L\{f(t)\}=\int_{0}^{\infty} f(t) e^{-s t} d t
$$

Em (8) a função $\mathrm{f}(t)$ é zero para $t<0$. Aplicando a transformada Inversa de Laplace (3) e (4) e considerando $s=j \omega$ são obtidas (9) e (10):

$$
\begin{aligned}
& I_{A}(t)=\frac{1}{2 \pi} \int_{-j \infty}^{+j \infty}\left[Y_{A A}(\omega) V_{A}(\omega)+Y_{A B}(\omega) V_{B}(\omega)\right] e^{j \omega t} d \omega \\
& I_{B}(t)=\frac{1}{2 \pi} \int_{-j \infty}^{+j \infty}\left[Y_{B A}(\omega) V_{A}(\omega)+Y_{B B}(\omega) V_{B}(\omega)\right] e^{j \omega t} d \omega
\end{aligned}
$$

As equações (9) e (10) geralmente de complexa resolução, pois dependem das condições de carga da linha que dificultam encontrar uma solução analítica para $\mathrm{I}_{\mathrm{A}}(t)$ e $\mathrm{I}_{\mathrm{B}}(t)$. Assim utiliza o método numérico para resolução das equações hiperbólicas (3) e (4) usando a transformada Inversa de Laplace proposta pelos autores [12-14], obtendo as tensões e correntes no domínio do tempo.

\section{B. MODELO A PARÂMETROS A DISCRETOS}

O modelo a parâmetros discretos, ou Lumped Parameters Model (LPM), é amplamente empregado para análise de transitórios eletromagnéticos em sistemas de potência. Nesse modelo a LT é representada por uma cascata de circuitos $\pi$. A Fig. 2 mostra uma LT monofásica representada por uma quantidade genérica de circuitos $\pi$ em cascata e as equações de estado que descrevem as correntes e tensões da linha.

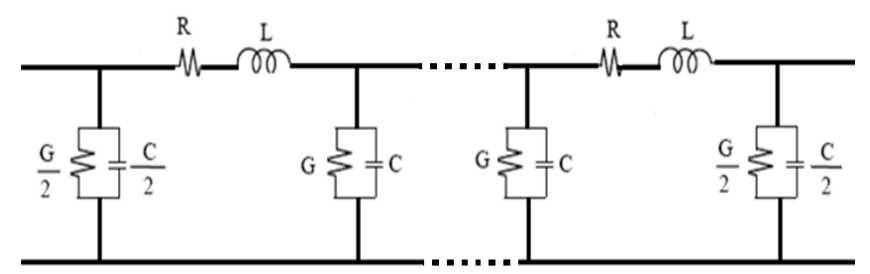

Figura 2. Linha de transmissão representada por cascata de circuitos $\pi$. 
Na Fig. 2, os parâmetros R e L são a resistência e indutância de cada circuito $\pi$ respectivamente. Os parâmetros $\mathrm{G}$ e $\mathrm{C}$ são a condutância e a capacitância de cada circuito $\pi$ respectivamente. Os parâmetros $\mathrm{R}, \mathrm{L}, \mathrm{G}$ e $\mathrm{C}$ são escritos como:

$$
R=R^{\prime} \frac{d}{n} \quad L=L^{\prime} \frac{d}{n} \quad C=C^{\prime} \frac{d}{n} \quad G=G^{\prime} \frac{d}{n}
$$

Em (11) os parâmetros longitudinais R' e L' e transversais parâmetros $\mathrm{C}^{\prime}$ e $\mathrm{G}^{\prime}$ são dados por unidade de comprimento, $d$ é o comprimento da linha e $n$ é o número de circuitos $\pi$ usado na representação da LT. Nesse modelo as correntes e tensões são obtidas diretamente no domínio do tempo [15-17]. Considerando a linha de transmissão de comprimento $d$, representada por $n$ circuitos $\pi$ como mostra a Fig. 2, as correntes e tensões são escritas como mostrado em (12).

$$
[\dot{x}(t)]=[A][x(t)]+[B] u(t)
$$

Em (12) $[x]$ é o vetor com correntes e tensões ao longo da cascata são escritos na forma:

$$
\begin{aligned}
& {[\dot{x}]=\left[\begin{array}{lllllll}
\dot{i}_{1}(t) & \dot{v}_{1}(t) & \dot{i}_{2}(t) & \dot{v}_{2}(t) & \ldots & \dot{i}_{n}(t) & \dot{v}_{n}(t)
\end{array}\right]^{T}} \\
& {[x]=\left[\begin{array}{lllllll}
i_{1}(t) & v_{1}(t) & i_{2}(t) & v_{2}(t) & \ldots & i_{n}(t) & v_{n}(t)
\end{array}\right]^{T}}
\end{aligned}
$$

As matrizes [A] e [B] são as matrizes de estado para LT representada pelo modelo a parâmetros discretos. As matrizes A e B dependem da configuração do terminal receptor da LT para sua formação. Assim por exemplo para uma linha terminada com uma impedância $Z_{C}$ no terminal receptor, representada por $n$ circuitos $\pi$, as matrizes [A] e [B] são dadas por:

$$
[\mathrm{A}]=\left[\begin{array}{cccccc}
-R / L & -1 / L & & & & \\
1 / C & -G / C & -1 / C & & & \\
& \ddots & \ddots & \ddots & & \\
& & \ddots & \ddots & \ddots & \\
& & & 1 / L & -R / L & -1 / L \\
& & & & 2 / C & -\left(G \cdot Z_{C}+2\right) / C \cdot Z_{C}
\end{array}\right]
$$

$$
[B]=\left[\begin{array}{llllll}
\frac{1}{L} & 0 & 0 & \cdots & 0 & 0
\end{array}\right]^{T}
$$

A matriz [A] é quadrada de ordem $2 n$ e [B] tem a dimensão $(2 n \times 1)$. A equação (12) será resolvida usando o método de Heun.

\section{FILTRO PASSA-BAIXA PASSIVO}

Um filtro passa-baixas passivo é um filtro que permite a passagem de sinais com frequência abaixo da frequência de corte estabelecida pelo filtro, reduzindo as amplitudes dos sinais com frequência acima da frequência de corte $\left(f_{c}\right)$. Foi observado que o espectro das oscilações espúrias apresenta componentes de elevadas frequências. Assim o filtro passabaixa proposto mitigará essas componentes, tornando as simulações obtidas mais próximas do valor real. O filtro é inserido diretamente no modelo a parâmetros discretos. Em
[18] os autores propõem um filtro digital do tipo FIR para reduzir as oscilações espúrias. Esse filtro reduziu consideravelmente as oscilações, mas apresenta a desvantagem de ser pós-processado, ou seja, é necessário similar, armazenar e em seguida aplicar o filtro digital no sinal desejado. Existem diversos filtros passa-baixas na literatura, mas nesse trabalho serão utilizados os filtros da Fig. 3.

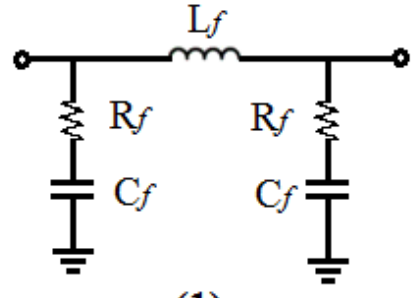

(1)

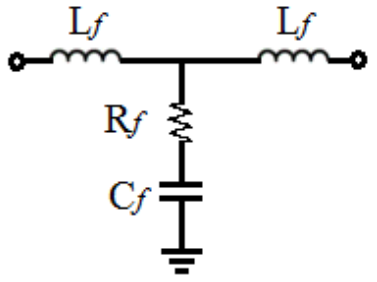

(2)
Figura 3. Filtros passa-baixas propostos para redução das oscilações espúrias.

Na Fig. 3 a configuração (1) será denominada de filtro PI e na configuração (2) será denominada de filtro T nas simulações. $\mathrm{Na}$ próxima seção serão apresentados os resultados de uma linha de transmissão representada pelo modelo a parâmetros discretos com e sem filtros passa-baixas inseridos.

\section{V.RESULTADOS}

Uma linha de transmissão monofásica será energizada por uma fonte senoidal no terminal emissor A e com uma carga resistiva $Z_{C}$ conectada no terminal receptor conforme mostra a Fig. 4. A linha será representada pelo modelo a parâmetros discretos, com 100 circuitos $\pi$ em cascada, cujo comprimento é de $100 \mathrm{~km}$ e energizada no instante $t=0$ e será calculada a tensão $V_{B}(t)$ para com e sem os filtros (1) e (2) comparadas com a resposta obtida pelo modelo ULM (resposta ideal).

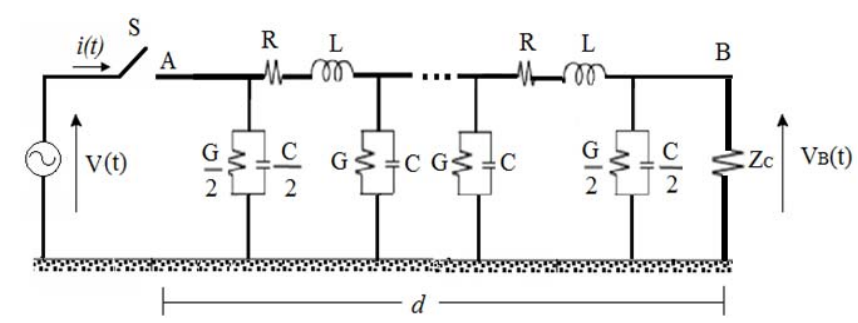

Figura 4. Linha monofásica representada pelo modelo a parâmetros discretos.

Na Fig. 4 os parâmetros elétricos da LT são dados pela tabela

TABELA I. PARÂMETROS DA LINHA DE TRANSMISSÃO.

\begin{tabular}{cc} 
PARÂMETROS & VALORES \\
\hline $\mathrm{R}$ & $0,05 \Omega / \mathrm{km}$ \\
$\mathrm{L}$ & $1,7 \mathrm{mH} / \mathrm{km}$ \\
$\mathrm{G}$ & $0,556 \mu \mathrm{S} / \mathrm{km}$ \\
$\mathrm{C}$ & $6,58 \mathrm{nF} / \mathrm{km}$ \\
$\mathrm{Z}_{\mathrm{C}}$ & $2000 \Omega$
\end{tabular}

Considerando que a linha será energizada por uma fonte senoidal dada por:

$$
\mathrm{V}(t)=V_{0} \cos (\omega t)
$$


Na equação (15) $V_{0}$ é a amplitude e $\omega$ é a frequência angular da onda senoidal. Para todas as simulações, tem-se que $V_{0}=100$ $\mathrm{kV}$ e $\omega=377 \mathrm{rad} / \mathrm{s}$ e os resultados serão apresentados em valores por unidade (p.u.), sendo $100 \mathrm{kV}$ o valor de base. A Fig. 5 mostra $\mathrm{V}_{\mathrm{B}}(t)$ para LT da Fig. 4 com as respostas do ULM e do modelo a parâmetros discretos (LPM) com 100 circuitos $\pi$.

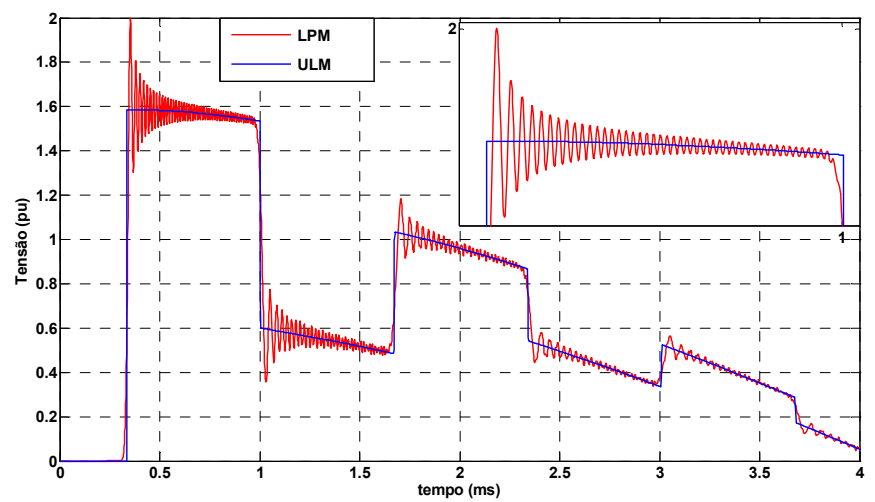

Figura 5. $V_{B}(t)$ para os modelos a parâmetros: discretos (vermelha) e distribuídos (azul).

As respostas apresentam o mesmo comportamento, mas as curva vermelha apresenta as oscilações espúrias, decorrentes da representação de um segmento de linha, cujos parâmetros são distribuídos, por elementos discretos de circuito. Essas oscilações não correspondem ao valor real da tensão. Assim considerando a primeira oscilação, tem-se que a curva vermelha apresenta um pico 30\% superior ao da curva azul. Esse pico, caso considerado, pode resultar na atuação indevida do sistema de proteção ou na superestimação dos isoladores da linha durante a fase de projeto. A Fig. 6 mostra a linha com um o filtro (1) conectado no terminal receptor da linha.

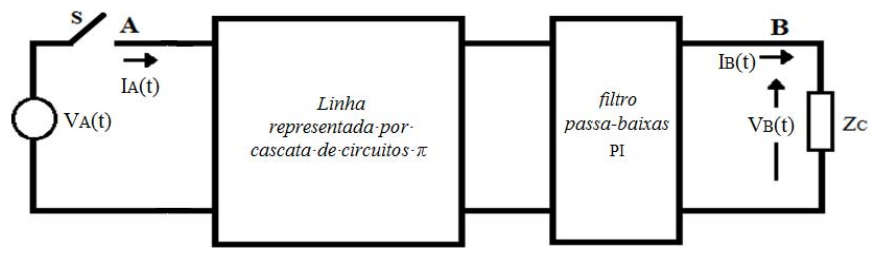

Figura 6. Filtro passa-baixas (1) inserido no modelo a parâmetros discretos.

Os parâmetros dos filtros passa-baixas (1) e (2) permaneceram invariáveis durante as simulações e são apresentados na tabela II.

Usando os parâmetros da tabela II, a frequência de corte é igual a $43,7 \mathrm{kHz}$. Assim as componentes acime desse valor serão reduzidas significativamente. Esse valor foi escolhido uma vez observado o espectro de frequência das oscilações espúrias

TABELA II. PARÂMETROS DO FILTRO PASSA-BAIXAS.

\begin{tabular}{cc}
$\begin{array}{c}\text { PARÂMETROS } \\
\text { DO FILTROS }\end{array}$ & VALOR \\
\hline $\mathrm{R}_{f}$ & $900 \Omega$ \\
$\mathrm{L}_{f}$ & $5,4 \mathrm{mH}$ \\
$\mathrm{C}_{f}$ & $8,4 \mathrm{nF}$
\end{tabular}

A frequência de corte para do filtro (1) e (2) é dada por (16):

$\left.f_{C}=\frac{1}{2 \pi} \sqrt{\frac{\left(R_{f}^{2} C_{f}^{2}-2 L_{f}^{2} C_{f}^{2}\right)+\sqrt{\left(R_{f}^{2} C_{f}^{2}-2 L_{f}^{2} C_{f}^{2}\right)^{2}+4 L_{f}^{2} C_{f}^{2}}}{2 L_{f}^{2} C_{f}^{2}}}\right)$

Uma vez definida a frequência de corte a ser projetada, os parâmetros $\mathrm{R}_{f}, \mathrm{C}_{f}$ e $\mathrm{R}_{f}$ do filtro foram ajustados para se obter esse valor conforme (16).

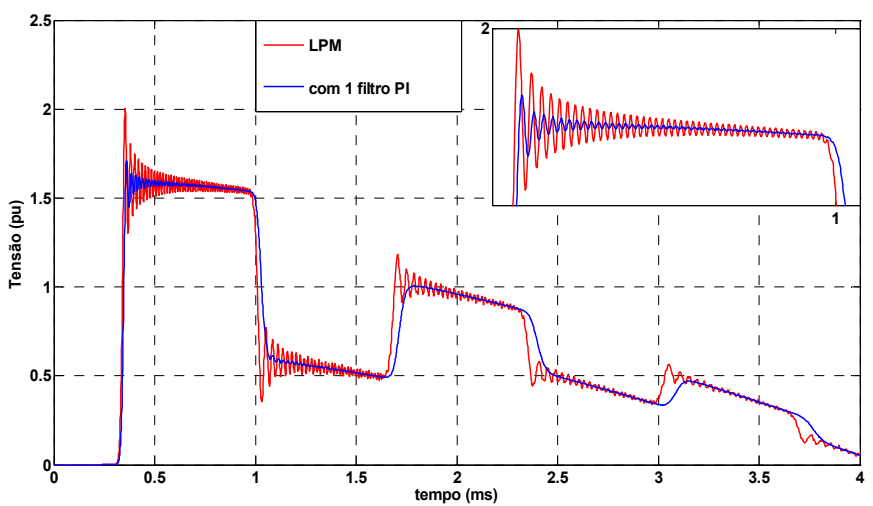

Figura 7. $V_{B}(t)$ para o modelo a parâmetros discretos com filtro (curva azul) e sem filtro (curva vermelha).

A Fig. 7 mostra $V_{B}(t)$ para a LT da Fig. 6. As oscilações espúrias foram consideravelmente reduzidas com o filtro inserido. Em detalhe a primeira reflexão do transitório onde se observa a redução das oscilações, porém a um pequeno deslocamento na resposta devido ao deslocamento de fase inerente do filtro passa-baixas. A Fig. 8 mostra o espectro de frequência para as curvas da Fig. 7 usando a transformada de Fourier. As amplitudes considerando o modelo a parâmetros discretos com o filtro passa-baixas foram reduzidas (curva azul). As componentes de elevada frequência foram atenuadas, reduzindo as amplitudes das oscilações espúrias nas simulações no domínio do tempo.

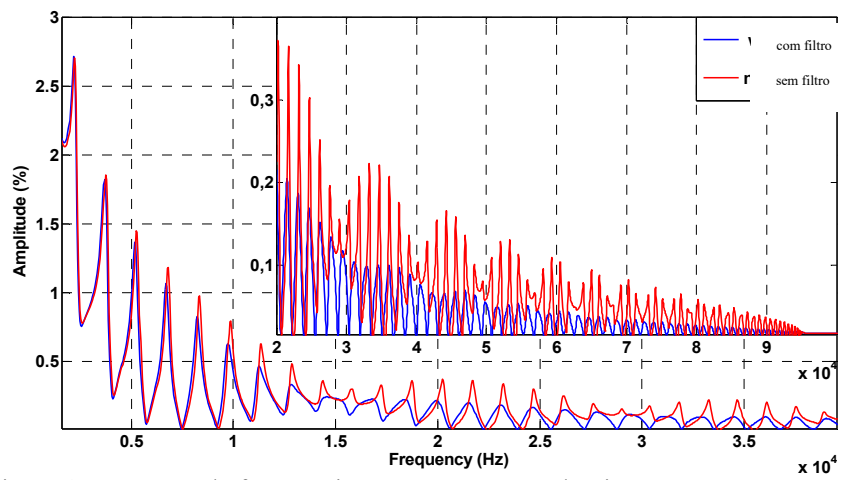

Figura 8. Espectro de frequência para as respostas da Fig. 7.

Outra possibilidade para reduzir as oscilações espúrias consiste em utilizar 2 filtros passa-baixas do tipo (1), um em cada terminal da linha, conforme mostra a Fig.9. 


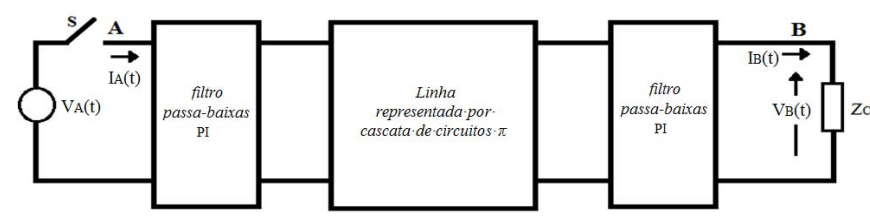

Figura 9. Linha de transmissão com 2 filtros passa-baixas PI no LPM.

A LT monofásica da Fig. 9 será representada por100 circuitos $\pi$ e 2 filtros passa- baixas. A Fig. 10 mostra $V_{B}(t)$ considerando a LT com e sem os filtros passa-baixas no LPM.

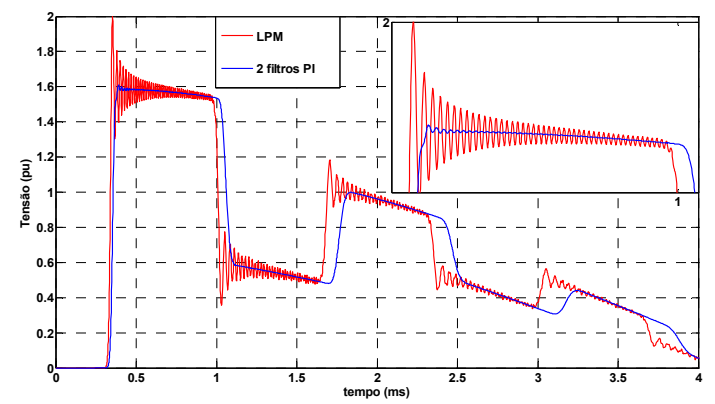

Figura 10. $V_{B}(t)$ com 2 filtros passa-baixas (azul) e sem filtro (vermelha).

Nessa condição, as oscilações apresentaram uma atenuação mais significativa quando comparadas com a Fig. 7, como pode ser visto no detalhe da primeira reflexão. Assim com 2 filtros as respostas se tornam mais próximas ao valor esperado A Fig. 11 mostra a mesma LT representada pelo modelo a parâmetros discretos com uma carga $Z_{C}$ e com 1 filtro passa-baixas do tipo (2) conectado em cascata com o ultimo circuito $\pi$ ao terminal receptor.

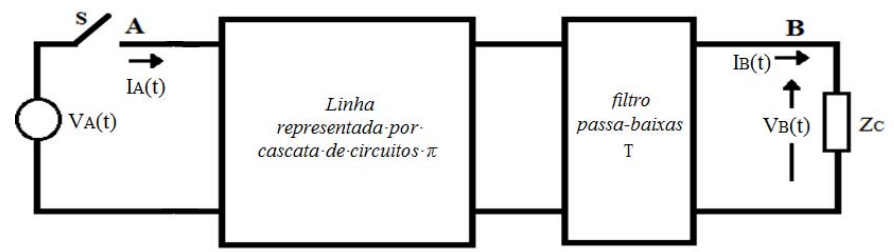

Figura 11. Linha de transmissão com filtro passa-baixas do tipo (2).

A Fig. 12 mostra a LT com 2 filtros filtros-baixas do tipo (2) inseridos .

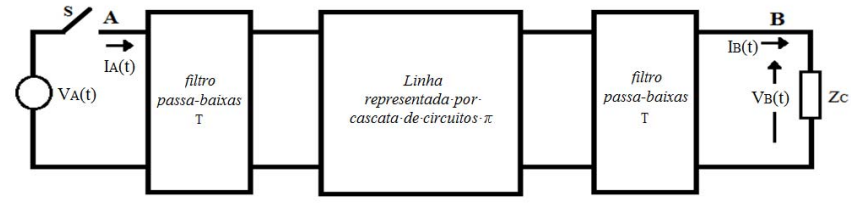

Figure 12. Transmission line with 2 low-pass filter (2).

Os circuitos das Fig.11 e 12 serão nomeados de "circuito (1)" e "circuito (2)" respectivamente para as próximas simulações. A Fig. 13 mostra $V_{B}(t)$ para a LT com 100 circuitos $\pi$ obtidas nos "circuito (1)" e "circuito (2)" e para o ULM.

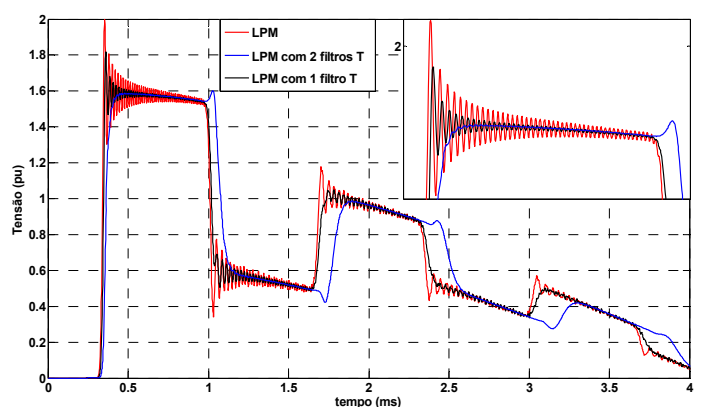

Figura 13. $\mathrm{V}_{\mathrm{B}}(\mathrm{t})$ para "circuito (1)" (azul), "circuito (2)"(preta) e ULM (vermelha).

A tensão $V_{B}(t)$ no circuito (1) apresenta pequenas oscilações enquanto que no circuito (2) elas são completamente reduzidas. Há um deslocamento observado, sendo que no circuito (2) esse deslocamento se torna expressivo. O modelo a parâmetros distribuídos não apresenta oscilações espúrias porque utiliza as equações hiperbólicas da linha no domínio da frequência. A fig. 14 mostra a LT representada pelo modelo a parâmetros destruídos de $100 \mathrm{~km}$ e energizada por uma fonte senoidal dada por (14) e no terminal receptor B está conectada uma carga $Z_{C}$.

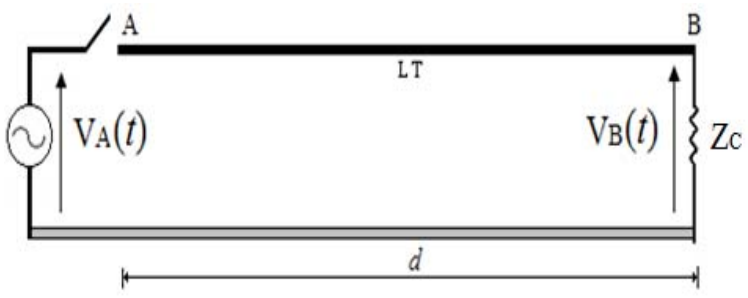

Figura 14. LT com uma carga resistiva no terminal receptor.

Os parâmetros da LT são dados pela tabela I. A resposta obtida com esse modelo, utilizando o ULM, será comparada com a resposta obtida com a LT representada pelo modelo a parâmetros discretos (LPM) com 2 filtros passa-baixas do tipo (1) da Fig.9. A Fig. 15 mostra as respostas obtidas no domínio do tempo.

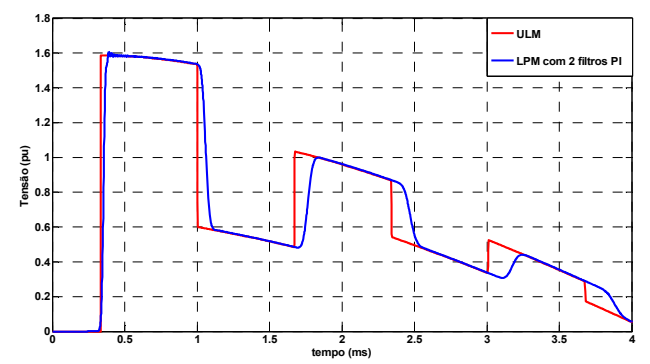

Figura 15. $\mathrm{V}_{\mathrm{B}}(\mathrm{t})$ com os modelos: distribuídos (vermelha) e discretos com filtro (azul) do tipo (1).

A Fig. 15 mostra a resposta para 2 filtro passa-baixas é similar a resposta obtida pelo modelo a parâmetros distribuídos usando ULM, porém o deslocamento é intrínseco ao filtro. Os filtros (1) e (2) reduziram as oscilações consideravelmente. A inserção de mais filtros passa-baixas é possível no modelo a parâmetros discretos, porém pode ocorrer uma saturação nas respostas obtidas e a defasagem será maior. As oscilações dependem diretamente da quantidade de circuitos $\pi$ e foi 
estabelecida uma quantidade de 1 circuito $\pi$ por quilometro nesse trabalho. Outro fato a ser enfatizado consiste que a frequência de corte é ajustável, assim é necessário conhecer o espectro de frequência e ajustar os parâmetros do filtro passabaixas. Assim as oscilações espúrias podem ser mais atenuadas e as respostas estarão mais próximas das obtidas pelo modelo a parâmetros distribuídos usando ULM.

\section{CONCLUSÕES}

Nesse trabalho foram apresentados dois modelos de linha de transmissão comumente utilizados para estudo de transitórios eletromagnéticos modelo a parâmetros discretos apresente oscilações espúrias que não representam o valor real das simulações. Assim para reduzir as oscilações espúrias foram projetados dois tipos de filtro passa-baixos passivos que foram inseridos diretamente no modelo a parâmetros discretos. Os resultados mostraram que com apenas um filtro inserido, as oscilações foram reduzidas significativamente Usando 2 filtros passa-baixas, as oscilações foram praticamente eliminadas, fazendo com que as respostas se tornassem semelhantes as obtidas pelo ULM, considerado exato, pois suas soluções provem das equações hiperbólicas da linha.

Pode-se também observar que a inserção de filtros passivos provoca um deslocamento nas respostas no domínio do tempo que devem ser consideradas para analise de transitórios, mas em regime permanente todas as respostas convergem para o mesmo valor. $O$ filtro passa-baixas apresenta a vantagem de ser inserido diretamente inserido no modelo a parâmetros discretos e desta forma a filtragem é feita em tempo real, principal característica do filtro passive quando comparado ao filtro digital. Outro fato consiste que o filtro pode ter sua frequência de corte ajustada utilizando apenas parâmetros. Assim a inserção de um filtro passa-baixas no modelo a parâmetros discretos mostrou ser uma alternativa válida para reduzir as oscilações espúrias, tornando-o mais preciso e confiável, e pode ser utilizado na análise de transitórios eletromagnéticos de sistemas de potência.

\section{AGRADECIMENTOS}

Os autotres agradecem pelo apoio da Fundação de Amparo à Pesquisa do Estado de São Paulo - FAPESP (2012/13857-4) e do Conselho Nacional de Desenvolvimento Científico e Tecnológico - CNPq.

\section{REFERÊNCIAS}

[1] Ametani A. "The History and Recent Trends of Transient Analysis in Transmission Lines," The IPST Conference, Vancouver, Canada,2013.

[2] Dommel, H. W. "EMTP Theory Book", Microtran Power System Analysis Corporation, Vancouver, British Columbia,1996.

[3] Mamis, M. S. "Computation of Electromagnetic Transients on Transmission Lineswit Nonlinear Components", IEE Proceedings Generarion, Transmission and Distribution, Vol. 150, No 2, pp. 200$203,2003$.

[4] Mamis, M. S., Nacaroglu A.,"Transient Voltage and Current Distributions on Transmission Lines", IEE Proceedings Generarion, Transmission and Distribution,Vol. 149, No 6, pp. 705-712,2012.

[5] Mácias, J. A. R., Expósito, A. G., Soler A. B. "A Comparison of Techniques for State-Space Transient Analysis of Transmission Lines",
IEEE Transactions on Power Delivery, Vol. 20, No 2, pp. 894$903,2005$.

[6] Nelms, R. M., Sheble G. B., Steve R. N., Grigsby L. L."Using a Personal Computer to Teach Power System Transients", IEEE Transactions on Power Systems, Vol. 4, No 3, pp. 1293-1297,1989.

[7] Chipman R. A., "Teoria e Problemas de Linhas de Transmissão", Editora Mc Graw-Hill do Brasil Ltda, São Paulo, SP,1976.

[8] Kurokawa S., Yamanaka F. N. R., et all. "Inclusion of the frequency effect in the lumped parameters transmission line model: State space formulation".Electric Power Systems Research, Vol. 79, issue 7, pp. $1155-1163,2009$.

[9] Araújo, A. R. J, Silva R.C., Kurokawa S. "Representation of Transmission Lines: Comparison of Models and Parameters Distributed Discrete Parameters". Revista IEEE América Latina, vol. 11, pp. 1047$1052,2013$.

[10] Kurokawa S., Yamanaka F. N. R., et all . "Inclusion of the frequency effect in the lumped parameters transmission line model: State space formulation".Electric Power Systems Research, Vol. 79, issue 7, pp. 1155-1163,2009.

[11] Gustavsen, B. "Validation of Frequency Dependent Transmission Line Models", IEEE Transactions on Power Delivery, Vol. 20, No 2, pp. 925-933, 2005.

[12] Moreno P., Ramirez A., "Implementation of the Numerical Laplace Transform: A Review", IEEE Transactions on Power Delivery, Vol. 23, No 4, pp. 2599-2609,2008.

[13] Moreno, P., Naredo J. L, Guardado J. L (2008). "Frequency domain transient analysis of electrical networks including non-linear conditions". International Journal of Electrical Power \& Energy Systems, vol. 27, n. 2,pp. 139-146,2008.

[14] Budner, A., "Introduction of frequency-dependent line parameters into an electromagnetic transients program". IEEE Trans. Power App. And Systems, New York, vol. PAS-89, n. 1, pp. 88-97,1970.

[15] Dommel, H. W. "Digital Computer of Electromagnetic Transients in Single and Mutiphase Networks", IEEE Transactions on Power Apparatus and Systems, Vol.PAS-88, No 4, pp. 388-399,2007.

[16] Ma S., Xu B., Bo Z., Klimberk A., "The Reaserch on Lumped Parameter Equivalent Circuit of Transmission Line" Advances in Power System Control, Operation and Management (APSCOM 2009), 8th International Conference, pp 1-5,2009.

[17] Mamis, M. S."Computation of Electromagnetic Transients on Transmission Lines with Nonlinear Components", IEE Proceedings Generarion, Transmission and Distribution, vol. 150, No 2,pp. 200203,2003

[18] Costa, E. C. M, Kurokawa, S., Shinoda A.A., Pissolato J.,"Digital filtering of oscillations intrinsic to transmission line modeling based on lumped parameters". International Journal of Electrical Power \& Energy Systems, vol. 44, pp. 908-915,2013.

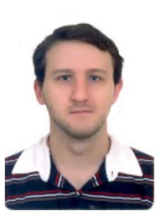

Anderson Ricardo Justo de Araújo, graduado (2012) e mestre (2014) em Engenharia Elétrica pela Faculdade de Engenharia Elétrica de Ilha Solteira, UNESP - Ilha Solteira e atualmente é estudante de doutorado em Engenharia Elétrica na Faculdade de Engenharia de Ilha Solteira, UNESP. Suas áreas de interesse são: transitórios eletromagnéticos em sistemas elétricos de potência utilizando métodos numéricos e modelagem de linhas de transmissão.

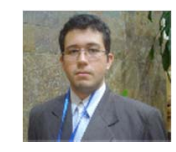

Rodrigo Cleber da Silva, graduado (2010) em mestre em engenharia elétrica pela Faculdade de Engenharia Ilha Solteira. Atualmente está no doutorado na mesma universidade. Professor no Instituto Federal de São Paulo (IFSP). Suas principais áreas de interesse são transitórios eletromagnéticos em sistemas de potência, modelos matemáticos para linhas de transmissão e métodos numéricos para análise transitória.

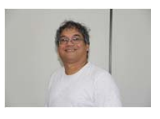

Sérgio Kurokawa (S'01-M'04), graduado em Engenharia Elétrica (1990). Desde 1994 atua como Professor na Faculdade de Engenharia de Ilha Solteira da Universidade Estadual Paulista (UNESP). Obteve o título de Doutor em Engenharia Elétrica na Faculdade de Engenharia Elétrica e da Computação da Universidade Estadual Paulista (UNICAMP). Suas principais áreas de interesse são transitórios eletromagnéticos em sistemas elétricos de potência e modelos de linhas de transmissão para simulações de transitórios eletromagnéticos em sistemas de potência. 